\title{
Differential Susceptibility to Synaptic Plasticity Reveals a Functional Specialization of Ascending Axon and Parallel Fiber Synapses to Cerebellar Purkinje Cells
}

\author{
Robert E. Sims and Nicholas A. Hartell \\ Department of Pharmacology, The School of Pharmacy, University of London, London WC1N 1AX, United Kingdom
}

Granule cell axons, via their parallel fibers, form synapses with Purkinje cells across large areas of the cerebellar cortex. Evidence for uniform transmission along parallel fibers to Purkinje cells is controversial, however, leading to speculation that the ascending axonal segment plays a dominant role in cerebellar processing. We have compared the relative susceptibilities of ascending axon and parallel fiber synaptic inputs to several forms of synaptic plasticity. We demonstrate that ascending axon synapses have a limited capability to undergo forms of long-term depression and potentiation compared with parallel fiber synapses. These results demonstrate that these two segments of the same axon play fundamentally different roles in cerebellar signaling, and, as such, the synapses formed between granule cells and Purkinje cells should not be treated as a homogenous population.

Key words: cerebellum; long-term depression; long-term potentiation; synaptic transmission; granule cell; Purkinje cell

\section{Introduction}

Long-term plasticity at synapses is thought to represent a cellular substrate for information storage in the brain, but it also provides a dynamic mechanism by which to sculpt patterns of information flow across neuronal structures. In the cerebellum, excitatory afferent information from mossy fibers is transmitted via granule cells (GCs) to Purkinje cells (PCs). GC axons ascend into the molecular layer of the cortex, where they bifurcate to form parallel fibers (PFs), which extend for several millimeters in either direction, forming one or at most two synapses with a large number of PCs (Napper and Harvey, 1988). This characteristic architecture has formed the basis for models of cerebellar function in which information is thought to be readily disseminated across the cortex along beams of parallel fibers. Evidence that information is reliably transmitted along the entire length of parallel fibers to PCs is, however, controversial. Whereas direct parallel fiber activation can spread some distance along fibers (Garwicz and Andersson, 1992; Vranesic et al., 1994; Heck, 1995), mossy fiber stimulation activates only patches of PCs directly above the mossy fiber termination zone (Eccles et al., 1972; Bower and Woolston, 1983; Cohen and Yarom, 1998) with little evidence of synchronous parallel fiber activation (Jaeger, 2003). These observations have revitalized a hypothesis in which synapses formed by the ascending segment of the GC axon, which represent $20 \%$ of the total GC input to PCs (Gundappa-Sulur et al., 1999), provide the principal excitatory drive to the cerebellar cortex (Llinás, 1982;

\section{Received Sept. 28, 2005; revised March 15, 2006; accepted March 15, 2006.}

This work was supported by the Biotechnology and Biological Sciences Research Council and the Royal Society. Correspondence should be addressed to Dr. Nicholas A. Hartell, Department of Pharmacology, The School of Pharmacy, University of London, 29-39 Brunswick Square, Bloomsbury, London WC1N 1AX, UK. E-mail: nicholas.hartell@pharmacy.ac.uk.

DOI:10.1523/JNEUROSCI.4121-05.2006

Copyright $\odot 2006$ Society for Neuroscience $\quad$ 0270-6474/06/265153-07\$15.00/0
Santamaria et al., 2002). Ascending axon synapses release transmitter with a higher mean probability than those formed by parallel fibers and display larger postsynaptic responses to quantal release events (Sims and Hartell, 2005). Furthermore, whereas up to $85 \%$ of synapses formed by parallel fibers appear to be electrically silent, this proportion is much reduced at ascending axon synapses (Isope and Barbour, 2002). These synaptic specializations confer each segment of the granule cell axon with fundamentally different transmission characteristics and suggest that they serve functionally different roles in cerebellar processing. To test the hypothesis that parallel fibers play a more modulatory role in cerebellar information flow whereas ascending axons are "hard-wired," we have compared the extent to which synapses formed by each axonal segment undergo various forms of plasticity associated with motor learning.

\section{Materials and Methods}

Slice preparation. Coronal, cerebellar slices ( $250 \mu \mathrm{m}$ thick) were obtained from the vermis of 14 - to 21 -d-old, male Wistar rats, using a cutting solution consisting of the following (in $\mathrm{mm}$ ): 250 sucrose, $2.5 \mathrm{KCl}, 26$ $\mathrm{NaHCO}_{3}, 1.25 \mathrm{NaH}_{2} \mathrm{PO}_{4}, 2 \mathrm{CaCl}_{2} \cdot 2 \mathrm{H}_{2} \mathrm{O}, 1 \mathrm{MgCl}_{2} \cdot 6 \mathrm{H}_{2} \mathrm{O}, 10$ glucose. Slices were then incubated for $1 \mathrm{~h}$ at room temperature in artificial CSF (aCSF) containing the following (in $\mathrm{mm}$ ): $120 \mathrm{NaCl}, 2.7 \mathrm{KCl}, 2.5$ $\mathrm{CaCl}_{2} \cdot 2 \mathrm{H}_{2} \mathrm{O}, 25 \mathrm{NaHCO}_{3}, 1.2 \mathrm{NaH}_{2} \mathrm{PO}_{4}, 1.2 \mathrm{MgSO}_{4} \cdot 7 \mathrm{H}_{2} \mathrm{O}$, and $11 \mathrm{glu}-$ cose, equilibrated with $95 \% \mathrm{O}_{2}-5 \% \mathrm{CO}_{2}$ to $\mathrm{pH}$ 7.4. Picrotoxin $(20 \mu \mathrm{M})$ was present in the aCSF to block transmission via $\mathrm{GABA}_{\mathrm{A}}$ receptors.

Electrophysiology. Slices were transferred to a chamber on an upright microscope and perfused with aCSF at a rate of $1.5-2 \mathrm{ml} \mathrm{min}^{-1}$ at room temperature. Whole-cell patch-clamp recordings were made from the somata of PCs $20-100 \mu \mathrm{m}$ below the surface of the slice. For recording, patch electrodes $(3-5 \mathrm{M} \Omega$ ) were filled with solutions containing the following (in mM): $132 \mathrm{~K}$-gluconate, $8 \mathrm{NaCl}, 2 \mathrm{MgCl}_{2} \cdot 6 \mathrm{H}_{2} \mathrm{O}, 30 \mathrm{HEPES}$, $4 \mathrm{Na}_{2} \mathrm{ATP}, 0.3 \mathrm{GTP}$, and 0.5 EGTA or 0.5 BAPTA. In some cases, the concentration of BAPTA was raised to $10 \mathrm{~mm}$ to reduce the likelihood of concurrent postsynaptic long-term depression (LTD) induction. At least 
15 min were allowed to elapse after entering whole-cell mode before the start of data collection to enable sufficient diffusion of BAPTA.

In a coronal slice orientation, the T-shaped structure of GC axons is preserved, allowing a relatively selective activation of parallel fiber and ascending axon synapses to the same PC (Sims and Hartell, 2005). Parallel fiber synapses were activated by stimulating through aCSF-filled patch electrodes, with resistances of $1-2 \mathrm{M} \Omega$, placed midway between the PC layer and pia mater to a depth of 50-100 $\mu \mathrm{m}, 150-300 \mu \mathrm{m}$ lateral to the recorded PC. This input will subsequently be referred to as the parallel fiber pathway. Ascending axon synapses were activated with electrodes placed in the GC layer, $30-100 \mu \mathrm{m}$ directly behind the PC soma. Ascending axons make $\sim 30$ synaptic contacts before forming parallel fibers (Pitchitpornchai et al., 1994). Because parallel fibers make on average only one or at most two synapses with a single PC (Harvey and Napper, 1991), then in this configuration, the likelihood of activating parallel fiber synapses is small. Although unlikely, we cannot rule out, however, a potential contribution from synapses formed by the parallel fiber very close to the point of bifurcation. With this caveat, this input will subsequently be referred to as the ascending axon pathway. To test whether a difference in the density of synapse activation by ascending axon and parallel fiber pathways might influence the induction of plasticity, a series of experiments was performed in which a stimulation electrode was placed in the GC layer, 150-300 $\mu \mathrm{m}$ lateral to the recorded $\mathrm{PC}$ at least $50 \mu \mathrm{m}$ from the PC layer. In this configuration, because ascending axons rise to different levels within the molecular layer, GC activation produces a more dispersed activation of synapses to the recorded PC. Placement here also eliminates any possibility of retrograde activation of ascending axon segment synapses. In all experiments, baseline stimulus intensities were carefully adjusted to elicit relatively small synaptic currents to ensure the specificity of synaptic input pathway and to standardize, as closely as possible, the number of contributing synapses in each pathway. Stimulation strengths did not exceed $20 \mathrm{~V}$ and were applied for $100 \mu \mathrm{s}$.

Whole-cell recordings were made with an Axopatch 200B; signals were filtered at $5 \mathrm{kHz}$ (8-pole Bessel filter) and digitized at $10 \mathrm{kHz}$. In all experiments, series resistances (5-20 M $\Omega$ ) were compensated (>75\%) and monitored throughout. Experiments were terminated if series resistance, input resistance, or holding current changed significantly during the experiment. Data were collected using "The LTP program" (Anderson and Collingridge, 1999).

The effects of five different protocols previously reported to produce either long-term potentiation (LTP) or LTD were compared at parallel fiber and ascending axon pathways. In all cases, a 10 min period of baseline stimulation was initially applied during which pairs of pulses (50 ms interval) were delivered alternately to parallel fiber and ascending axon pathways at a rate of 0.1 or $0.2 \mathrm{~Hz}$. Two forms of long-term depression were examined. Because LTD requires the postsynaptic elevation of calcium, an intracellular solution containing $0.5 \mathrm{~mm}$ EGTA was used and induction protocols were performed in current-clamp mode. For conjunctive LTD $\left(\operatorname{LTD}_{\mathrm{CJ}}\right)$, cells were held between -65 and $-70 \mathrm{mV}$ and one of the pathways was stimulated five times at $100 \mathrm{~Hz}$, followed $100 \mathrm{~ms}$ later by stimulation of the climbing fiber (CF) input. This protocol was repeated 50 times at $2 \mathrm{~s}$ intervals. To test for CF-independent LTD, the intensity of stimulation to one pathway was raised to elicit EPSPs $>10$ $\mathrm{mV}$ and the frequency of stimulation raised to $1 \mathrm{~Hz}$ for $5 \mathrm{~min}$ (Hartell, 1996). Care was taken to avoid PC spiking. Stimulation parameters to the other pathway were unaltered. After the induction period, cells were returned to voltage-clamp mode. During the induction of LTD, the membrane resistance often decreased transiently but recovered to baseline levels over the subsequent $5-10 \mathrm{~min}$. Experiments were abandoned if the membrane resistance did not recover. Transient decreases in membrane resistance led to apparent differences in the onset rate of depression (for example, compare Figs. $1 b, 5 b$ ). Statistical comparisons were therefore only made when the membrane resistance had fully recovered.

Three protocols reported to produce long-term potentiation were also examined. To induce LTP of postsynaptic origin $\left(\mathrm{LTP}_{\mathrm{POST}}\right), 300$ pulses were applied to one pathway at a rate of $1 \mathrm{~Hz}$. The other pathway was left unstimulated during this period. To induce LTP of presynaptic origin $\left(\mathrm{LTP}_{\mathrm{PRE}}\right)$, the frequency of stimulation to one pathway was raised to 16
$\mathrm{Hz}$ for $15 \mathrm{~s}$; the other pathway was not stimulated during this time. Finally, the effects of a 10 min application of the protein kinase A (PKA) activator forskolin, which is also thought to produce LTP through presynaptic actions, were also examined $\left(\mathrm{LTP}_{\text {FORSK }}\right)$. Stimulation conditions to either pathway were unaltered during applications. After all five protocols, baseline rates of stimulation were resumed for at least $30 \mathrm{~min}$.

Data analysis. Additional analysis was performed using customized procedures in IgorPro software (WaveMetrics, Lake Oswego, OR). The means of six consecutive sweeps per pathway were calculated every $30 \mathrm{~s}$, and data were expressed as percentages of mean baseline levels. Pairedpulse ratios were calculated as the mean of the second of paired pulses divided by the mean of the first to reduce inaccuracies caused by random fluctuation (Kim and Alger, 2001). To confirm the location of each type of synaptic plasticity, a form of coefficient of variation (CV) analysis was used (Bekkers and Stevens, 1990). This analysis allows an assessment of whether changes in response amplitude result from a change in the probability of release $(P)$, the number of contributing transmitter release sites $(N)$, or the postsynaptic quantal amplitude $(Q)$. The mean amplitude and the reciprocal of the square of the coefficient of variation $\left(\mathrm{CV}^{-2}\right)$ of $30-50$ individual responses, sampled during a period of stable baseline recording conditions and 20-30 min after each plasticity-induction protocol, were calculated. The ratios of the second to the first values were calculated to obtain a value relative to baseline conditions, and normalized values of $\mathrm{CV}^{-2}$ were plotted against normalized amplitude. The mean amplitude of EPSCs represents the product of $N, P$, and $Q(N P Q)$. $\mathrm{CV}^{-2}$ is proportional to $N P / 1-P$. Therefore, a change in $Q$ is independent of $\mathrm{CV}^{-2}$, whereas a change in $N$ will be directly proportional to amplitude and $\mathrm{CV}^{-2}$. The Mann-Whitney $U$ test was used for statistical analysis between independent sample groups, and the Wilcoxon signed ranks test was used to compare differences between pathways to the same cell. Unless stated otherwise, all compounds were obtained from Sigma (St. Louis, MO).

\section{Results}

Several presynaptic and postsynaptic forms of long-term plasticity have been described at GC-PC synapses that could help to establish or maintain specialization of granule cell axon synapses. However, because no distinction between ascending and parallel fiber synapses has previously been made, the relative susceptibilities of each of these segments to cerebellar plasticity are not known. We set out, therefore, to establish whether synapses formed by the two segments of the granule cell axon were differentially susceptible to each of the forms of plasticity so far described between GCs and PCs.

\section{Long-term depression at ascending axon and parallel fiber synapses}

Cerebellar LTD is thought to result from the downregulation of postsynaptic AMPA receptors (Wang and Linden, 2000). Because this would effectively reduce the mean quantal amplitude and because parallel fiber synapses have lower mean quantal amplitudes than those formed by ascending axons (Sims and Hartell, 2005), we compared the extent to which each synaptic pathway to single PCs underwent two well characterized forms of LTD. Combining CF activation with parallel fiber stimulation, using a protocol designed to replicate physiological patterns of GC and climbing fiber activity (LT$\mathrm{D}_{\mathrm{CJ}}$ ), produced LTD of parallel fiber pathway EPSCs but not ascending axon responses. No depression was observed in either pathway when the CF was activated in association with ascending axon inputs (Fig. 1b,c; Table 1). Ascending axon synapses are generally formed on smaller diameter, more distally situated dendrites (Gundappa-Sulur et al., 1999). In the event that their position places them outside the influence of the CFmediated calcium transient, one of the requisite elements for LTD, we also tested the efficacy of another form of LTD that does 
a

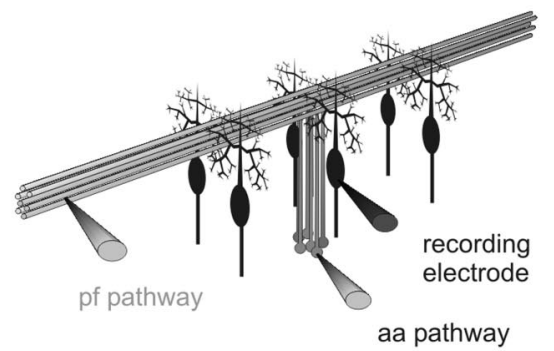

b

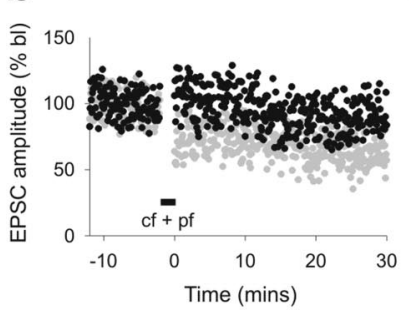

d
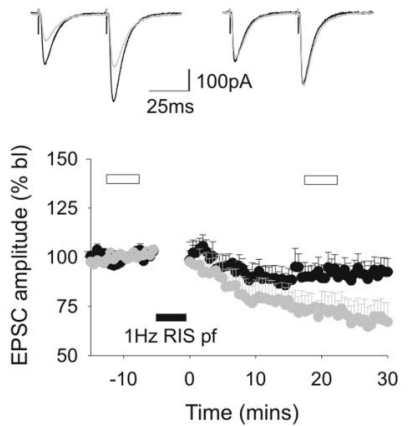

f

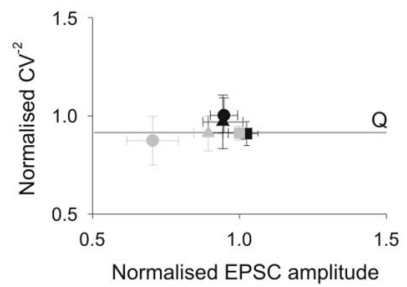

g

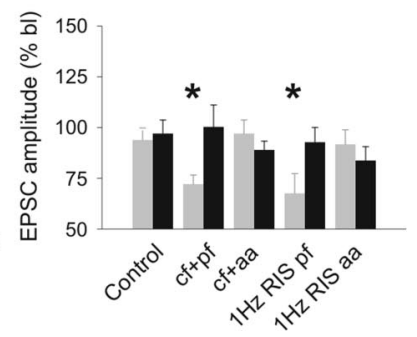

Figure 1. Ascending axon synapses to Purkinje cells are resistant to long-term depression. $\boldsymbol{a}$, The positioning of stimulating and recording electrodes in the granule cell and molecular layers for selective activation of ascending axon and parallel fiber synapses to Purkinje cells is shown. The effects of conjunctive activation of the (F input with parallel fiber ( $\boldsymbol{b}$ ) and ascending axon (c) pathway stimulation are shown. $\boldsymbol{d}$ and e illustrate the effects of $1 \mathrm{~Hz}$ raised intensity stimulation (1 Hz RIS) applied to the parallel fiber ( $n=10)$ and ascending axon pathways $(n=8)$, respectively. The means and SEs are shown. Shown superimposed above are the means of six consecutive sweeps sampled before (darker traces) and 20 min after (lighter traces) the period of $1 \mathrm{~Hz}$ RIS for each pathway. Responses to parallel fiber activation are shown on the lefthand side and ascending axon responses are shown to the right. $\boldsymbol{f}$ shows the relative changes in $\mathrm{CV}^{-2}$ values plotted against relative changes in mean EPSC amplitudes for each pathway with SEs before and 20 min after climbing fiber-independent LTD. Square symbols represent time-matched control data in which both pathways were activated alternately at $0.2 \mathrm{~Hz}$ throughout. $\boldsymbol{g}$, The means and SEs of parallel fiber (gray) and ascending axon pathway EPSC amplitudes (black) sampled 30 min after control stimulation are shown, along with those after pairing each pathway with the climbing fiber input or after presentation of the climbing fiber-independent LTD protocol. The asterisks indicate significant differences between pathways (Wilcoxon matched pairs test; $p<0.05$ ). Each data set represents a minimum of six separate experiments. In all panels, gray and black hues represent parallel fiber and ascending axon responses, respectively. Closed circles indicate when the conjunctive stimulation was applied to the parallel fiber pathway, and closed triangles represent the ascending axon pathway. bl, Baseline; aa, ascending axon; cf, climbing fiber; pf, parallel fiber. not require simultaneous CF activation (Hartell, 1996), referred to here as CF-independent LTD (Fig. 1d,e) (for details, see Materials and Methods). In this model, intracellular calcium levels are raised by increasing the strength of stimulation to the GC input for the period of induction. Application of this protocol to the parallel fiber pathway led to a selective depression of parallel fiber responses compared with time-matched control experiments in which both pathways were activated alternately at baseline rates and stimulus intensities throughout. No depression of ascending axon pathway responses was observed. Paired-pulse ratios did not change significantly in either pathway. When the induction protocol was applied to the ascending axon pathway, ascending axon synaptic responses initially increased slightly in amplitude but then returned to baseline levels. A small depression emerged after $30 \mathrm{~min}$, but this was not significantly different from control data. No depression of parallel fiber responses was observed under these conditions.

We next undertook an analysis of response variability before and after CF-independent LTD induction to help establish the site of origin of any long-term changes in synaptic strength. Surprisingly, because LTD is thought to be a postsynaptic process, CF-independent depression at the parallel fiber pathway was accompanied by a small increase in response variability, seen as a reduction in $\mathrm{CV}^{-2}$ values. We therefore examined the synaptic variability of our control data where ascending axon and parallel fiber pathways were activated at baseline intensities and rates for $30-40$ min. As seen in Figure $1 f$, similar decreases in $\mathrm{CV}^{-2}$ values were observed in the absence of synaptic depression, indicating that the increase in variability over time is a feature of prolonged recordings under these experimental conditions rather than synaptic depression itself. Accordingly, when compared with control data, we can see that the depression observed at parallel fiber synapses in response to parallel fiber stimulation occurs without any relative change in response variability and so reflects a decrease in quantal amplitude $(Q)$ (i.e., it is of postsynaptic origin).

\section{Postsynaptic long-term potentiation in ascending axon and parallel fiber synapses}

Recently, a variant of LTP has been reported at GC-PC synapses that can be induced by $1 \mathrm{~Hz}$ stimulation of GC-PC synapses, at baseline strengths, for $5 \mathrm{~min}$. This LTP is expressed postsynaptically (Lev-Ram et al., 2002) and is capable of reversing LTD (LevRam et al., 2003; Coesmans et al., 2004). Because postsynaptic LTP could result in an increase in $Q$, we examined the effects of 1 $\mathrm{Hz}$ stimulation to ascending axon and parallel fiber synapses in turn. In these experiments, $10 \mathrm{~mm}$ BAPTA was included in the patch pipette to prevent concurrent LTD expression and the baseline stimulation rate was lowered to $0.1 \mathrm{~Hz}$ to reduce run up of synaptic responses.

The application of $1 \mathrm{~Hz}$ stimulation to parallel fiber pathway inputs to PCs produced a long-lasting enhancement of parallel pathway EPSC amplitudes but not ascending axon pathway responses, which remained at baseline levels (Fig. 2a). Although parallel fiber potentiation was initially accompanied by a transient decrease in the paired-pulse ratio, this returned to baseline levels within $15 \mathrm{~min}$. The same stimulus protocol applied to the ascending axon pathway failed to produce potentiation (Fig. 2b). Ascending axon EPSC amplitudes remained indistinguishable from baseline levels or from time-matched control experiments. Interestingly, parallel fiber pathway responses, which were not evoked during induction of the ascending axon input, showed an immediate depression in five of the seven cells recorded that reached $79.3 \pm 9.8 \%$ of baseline levels within $20 \min (n=7)$. 
This was unexpected given the lack of any form of plasticity at the ascending axon input. The experiments were therefore repeated but stimulation to the parallel fiber pathway was maintained at baseline rates during $1 \mathrm{~Hz}$ stimulation of the ascending axon input. Under these conditions, EPSC amplitudes did not decline (Fig. $2 c$, Table 1). Therefore, the lack of synaptic input during ascending axon stimulation was responsible for depression at parallel fiber synapses. CV analysis was also performed for these experiments and compared with control data as before (Fig. $2 d$ ). This revealed that the long-term potentiation observed in the molecular layer pathway after $1 \mathrm{~Hz}$ stimulation did indeed appear to be the result of an increase in $Q$, confirming a postsynaptic effect. CV analysis suggests that the depression that emerged at parallel fiber pathways when left unstimulated may have been as a result of a reduction in the number of contributing release sites, because amplitude decreased proportionally with $\mathrm{CV}^{-2}$.

\section{Presynaptic long-term potentiation in ascending axon and parallel fiber synapses}

Higher frequencies of activation $(4-16 \mathrm{~Hz})$ also produce a form of LTP (Shibuki and Okada, 1992; Salin et al., 1996; Jacoby et al., 2001) that is accompanied by a change in the paired-pulse ratio, suggesting that it is caused by presynaptic events leading to an increase in release probability. When tested on parallel fiber pathway responses, $16 \mathrm{~Hz}$ stimulation elicited a potentiation of parallel fiber pathway responses (Fig. 3a). A small increase of ascending axon pathway responses also emerged but this was not significant compared with control data. The same protocol applied to the ascending axon pathway failed to produce potentiation in either pathway (Fig. $3 b$ ). Parallel fiber potentiation was accompanied by a reduction in paired-pulse ratio that was, in line with our previous results (Jacoby et al., 2001), significantly reduced for 10-15 min before recovering toward baseline levels $(p<0.05)$. A concurrent decrease in response variability (and therefore an increase in $\mathrm{CV}^{-2}$ ) over this time period further suggested a presynaptic site of action (Fig. $2 c-e$ ). Although not significant, $\mathrm{CV}$ analysis suggests that the small potentiation at the ascending axon pathway after raised frequency stimulation of the parallel fiber pathway may be postsynaptic in origin. Nevertheless, as in the case of the other forms of plasticity examined in this study, these experiments demonstrate that ascending axon inputs are less able to sustain plastic changes compared with parallel fiber inputs to the same cells.

Presynaptic LTP is dependent on PKA (Salin et al., 1996; Jacoby et al., 2001) and can be mimicked by application of the adenylate cyclase activator, forskolin. We therefore compared the effects of a $10 \mathrm{~min}$ application of forskolin $(10 \mu \mathrm{M})$ during $0.2 \mathrm{~Hz}$ alternate stimulation of the ascending axon and parallel fiber pathway (Fig. 4a). Twenty minutes after application, parallel fiber EPSC amplitudes were initially strongly potentiated. Ascending axon pathway responses also underwent an increase, but the peak level was much smaller and responses returned to baseline levels within $30 \mathrm{~min}$. $\mathrm{CV}^{-2}$ analysis demonstrated that potentiation in both pathways was presynaptic in agreement with previ- a
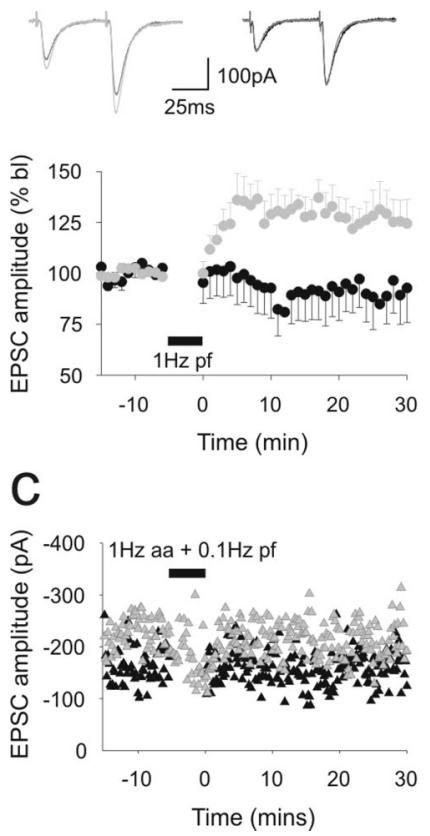

b
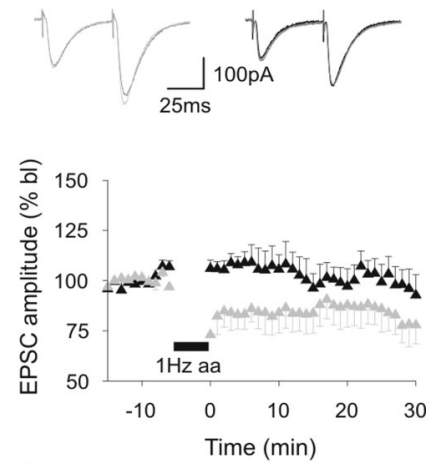

d

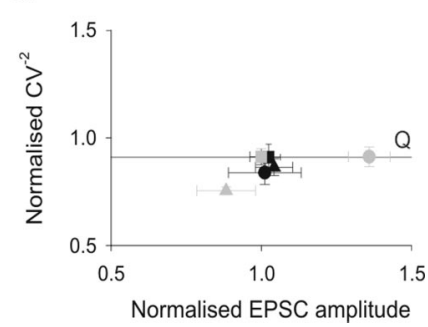

Figure 2. Ascending axon synapses to Purkinje cells are resistant to long-term potentiation. A comparison of the effect of applying $1 \mathrm{~Hz}$ stimulation for $5 \mathrm{~min}$ to parallel fiber (a) and ascending axon pathways to Purkinje cells. In each case, the alternate pathway was not stimulated during this period. Data are shown as in Figure 1, $d$ and $e$. Shown superimposed above are the means of six consecutive sweeps sampled before (darker traces) and 20 min after (lighter traces) the period of $1 \mathrm{~Hz}$ stimulation for each pathway. Responses to parallel fiber activation are shown on the lefthand side, and ascending axon responses are shown to the right. Gray and black symbols represent responses to parallel fiber and ascending axon pathway activation, respectively. In c, the parallel fiber pathway was activated at baseline rates while $1 \mathrm{~Hz}$ stimulation was applied to the ascending axon. dillustrates the changes in $\mathrm{CV}^{-2}$ that accompanied these stimulation protocols. bl, Baseline; aa, ascending axon; pf, parallel fiber. 
a
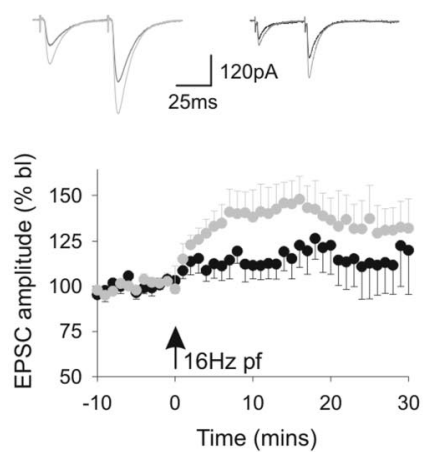

C
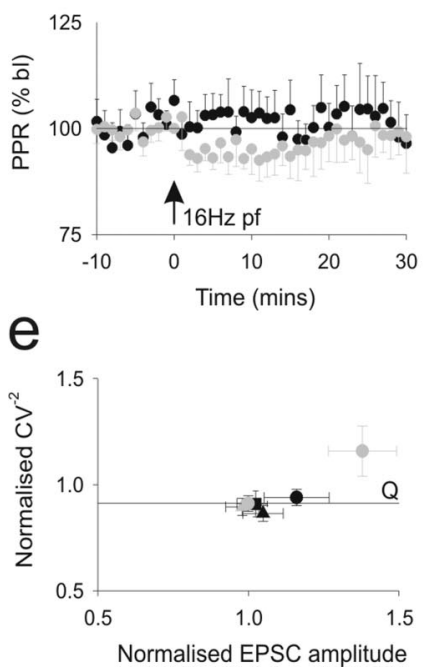

Figure 3. Ascending axon synapses to Purkinje cells are resistant to presynaptic potentiation. Bursts of stimuli were applied to parallel fiber $(\boldsymbol{a})$ and ascending axon $(\boldsymbol{b})$ pathway inputs to $\mathrm{PCs}$ at a rate of $16 \mathrm{~Hz}$ for $15 \mathrm{~s}$. The alternate pathway in each case was not stimulated during this period. The data are presented as in Figure 2, $a$ and $b$. The concomitant paired-pulse ratios are shown in $\mathbf{c}$ and $\boldsymbol{d}$. $\boldsymbol{e}$ illustrates the changes in $\mathrm{CV}^{-2}$ that resulted from these stimulation protocols. bl, Baseline; aa, ascending axon; pf, parallel fiber.

a

b
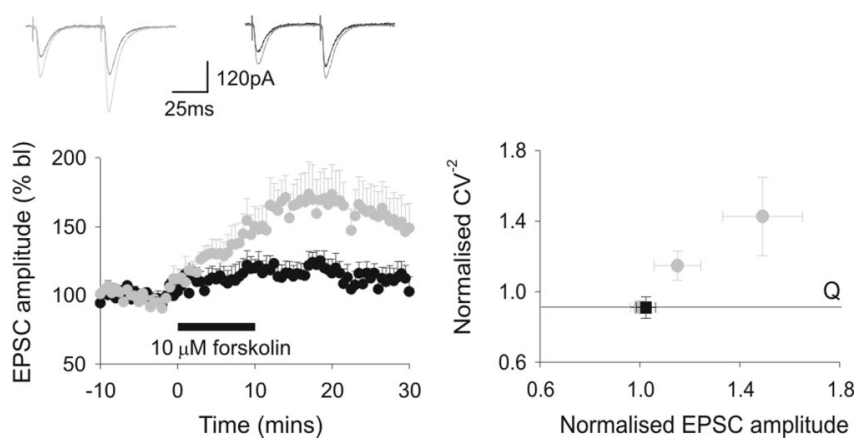

Figure 4. The effects of PKA activation on ascending axon and parallel fiber synaptic inputs to Purkinje cells. The effects of a $10 \mathrm{~min}$ application of $10 \mu \mathrm{m}$ forskolin on alternate activation of ascending axon and parallel fiber pathway responses are shown. The data are presented as in Figure 2, $a$ and $b$. Each pathway was activated at $0.2 \mathrm{~Hz}$ throughout. $\boldsymbol{b}$ illustrates the resulting changes in $\mathrm{CV}^{-2}$. ous observations (Salin et al., 1996; Jacoby et al., 2001). Because the increases in EPSC amplitudes were directly proportional to $\mathrm{CV}^{-2}$, this suggests that potentiation occurs through an increase in the number of contributing release sites.

\section{The influence of synaptic density on plasticity}

It has recently been suggested that stimulation of beams of parallel fibers causes an unphysiologically compact activation of localized regions of a PC, and through synaptic cross talk, this may increase the level of postsynaptic type 1 metabotropic glutamate receptor (mGluR1) activation and other consequential events including endocannabinoid inhibition of presynaptic glutamate release. Activation of mGluRs (Conquet et al., 1994; Hartell, 1994) and cannabinoid receptor type 1 are necessary for LTD (Safo and Regehr, 2005). We therefore examined the degree to which LTD and presynaptic and postsynaptic forms of LTP were expressed at PF synapses activated by stimulation of the GC layer, lateral to the recorded PC (Fig. 5a). Because ascending axons rise to different levels within the molecular layer, lateral GC activation produces a more dispersed activation of synapses to the recorded PC than direct parallel fiber activation. It also eliminates any possibility of retrograde activation of ascending axon synapses. For consistency and to allow proper comparison between experimental data sets, care was taken to ensure that the mean amplitudes of synaptic responses were identical to those produced by ascending axon or direct parallel fiber stimulation. Synaptic currents after lateral GC layer stimulation of the amplitudes used throughout this study do not produce synaptic cross talk, as evidenced by the absence of a slow, mGluR1-mediated current, even when activated at very high frequencies (Marcaggi and Attwell, 2005).

The results of these experiments are summarized in Figure 5 and Table 1. Pairing dispersed PF inputs with CF stimulation produced LTD (Fig. 5b). The level of depression was statistically indistinguishable from that resulting from direct PF stimulation. Similarly, $1 \mathrm{~Hz}$ raised intensity stimulation of the lateral GC layer also produced clear LTD. Both presynaptic and postsynaptic modes of LTP induction produced similar levels of potentiation to those emerging from direct PF stimulation (Fig. $5 d, e$ ). $\mathrm{CV}^{-2}$ analysis also showed that the origins of plasticity at dispersed PF inputs were identical to those produced by direct PF input. We conclude that, at the relatively low stimulus strengths used throughout this study, the pattern of synaptic input to PCs is unlikely to account for the difference in susceptibility to plasticity of ascending axon and parallel fiber inputs.

\section{Discussion}

The most striking observation from these results is that synapses supplied by the ascending segment of the GC axon to PCs are largely resistant to all of the forms of plasticity we tested compared with their parallel fiber counterparts. This provides compelling evidence that the two segments of the GC axon fulfill fundamentally different roles in cerebellar signal processing and adds an additional level of complexity to our knowledge of cerebellar signal processing.

The highly ordered morphology of the cerebellar cortex allows the independent and selective activation of ascending and parallel fibers segments of granule cell axons. Although the absolute degree of pathway selectivity produced by GC layer and molecular layer stimulation remains to be verified experimentally, it is sufficient to reveal clear differences in the transmission properties of synapses in each pathway (Sims and Hartell, 2005). Presynaptic and postsynaptic specialization at ascending axon and parallel fiber synapses confers each segment of the GC axon with distinct 

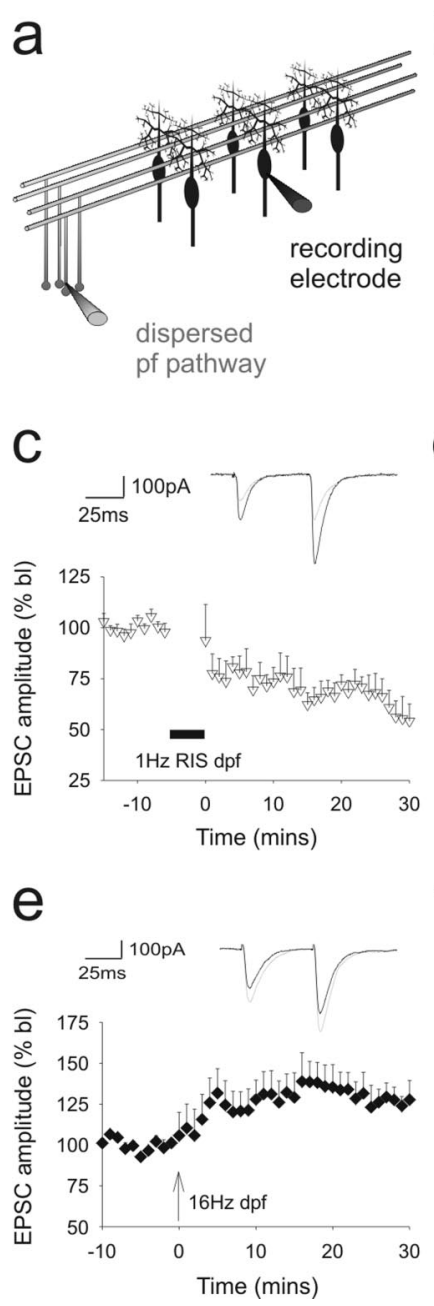

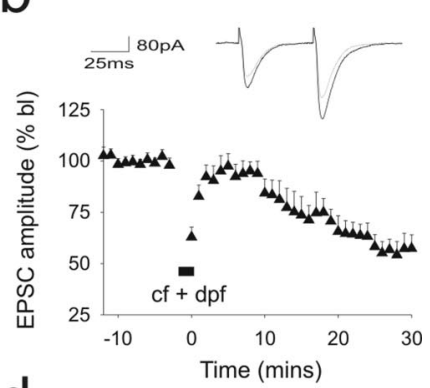

d
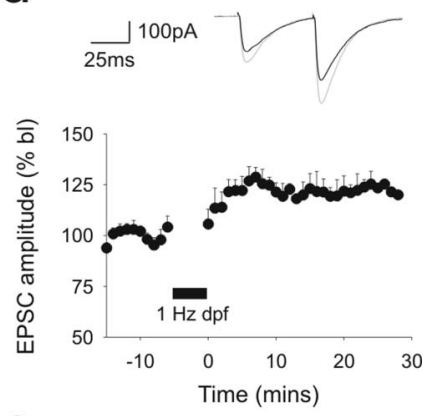

$f$

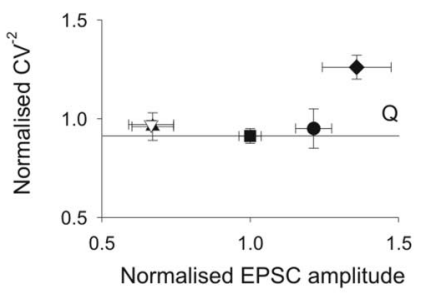

Figure 5. LTD and LTP do not depend on activation of beams of parallel fibers. $\boldsymbol{a}$ illustrates the arrangement of stimulating and recording electrodes for dispersed activation of parallel fibers. Because granule cell ascending axons rise to different levels within the molecular layer, stimulation of the granule cell layer 150-300 $\mu \mathrm{m}$ lateral to the recorded PC should produce a more dispersed pattern of parallel fiber activation compared with molecular layer stimulation. The effects of conjunctive activation of the $C F$ input with lateral $\mathrm{GC}$ stimulation $(\boldsymbol{b}), 1 \mathrm{~Hz}$ raised intensity stimulation (RIS) (c), $1 \mathrm{~Hz}$ stimulation (d), and $16 \mathrm{~Hz}$ stimulation of the lateral GC layer are shown (e) together with representative traces sampled before (black traces) and 20 min after (gray traces) each induction protocol. Pooled data represent the means and SEs of five to seven separate cells. $f$ illustrates the resulting changes in $\mathrm{CV}^{-2}$ for each protocol. The symbols match those used to display the results for each protocol in $\boldsymbol{a}-\boldsymbol{e}$. bl, Baseline; cf, climbing fiber; pf, parallel fiber; dpf, dispersed parallel fiber.

transmission properties. This enables GCs to transmit different types of information to PCs contacted by different sections of the axon according to their patterns of activity. We have previously argued that the relatively high synaptic release probability combined with multiple points of contact with a single PC enable ascending axon inputs to a PC to transmit low frequency information highly reliably, and, as such, they serve as event detectors to relay information within a single microzone that receives sensory information from a specific areas of the periphery. If this is the principal role of the ascending axon, then plasticity would serve little or no function and so the absence of any form of long-term plasticity at these synapses lends support to this notion.

Parallel fiber synapses have lower quantal amplitudes and release probabilities than those formed by ascending axons, and they can sustain short-term facilitation for longer, allowing them to transmit bursts of higher frequency information (Sims and Hartell, 2005). We now show that they can also readily undergo presynaptic and postsynaptic forms of long-term plasticity. Thus, their role is distinct from that of ascending axon synapses because they represent a pathway that is highly plastic and subject to dynamic regulation. Because they cross large areas of the cerebellar cortex, these properties provide parallel fibers with the capacity to pass information between microzones and undergo changes in synaptic strength in a direction that is determined by concurrent climbing fiber activity. In this way, parallel fiber inputs to PCs can be selectively dampened or strengthened and this establishes a pattern of communication between microzones that is based on learned experience.

The absence of plasticity at ascending axon synapses might indicate that they are intrinsically different from parallel fiber synapses and that they lack some of the cellular machinery necessary for plasticity. However, the limited ability of ascending axon synapses to undergo LTP in response to forskolin or $16 \mathrm{~Hz}$ stimulation might indicate that they have already undergone presynaptic potentiation and that this mechanism is saturated. This is an attractive hypothesis, because it explains their higher release probabilities compared with parallel fiber synapses.

Postsynaptic differences between ascending axon and parallel fiber synapses are less easily explained by the assumption that the two sets of synapses are mechanistically identical but simply exist in different plastic states. If this was the case, then one would expect both sets of synapses to be fully reversible, but this was clearly not the case. Therefore, we predict that there must be some intrinsic postsynaptic difference between the synapse types. A systematic evaluation of the presynaptic and postsynaptic elements at each synapse type is severely hampered by the difficulty in distinguishing between axonal segments at the ultrastructural level. Nevertheless, studies have revealed that there are presynaptic and postsynaptic morphological differences (GundappaSulur et al., 1999).

The relative positions of synapses formed by each segment of the GC axon could also influence the induction of plasticity. Ascending axon synapses are formed on smaller diameter, more distally situated dendrites (Gundappa-Sulur et al., 1999). This could conceivably place them outside the influence of the CF, which forms synapses on primary dendrites. If so, then the calcium signal supplied by CF activation may be insufficient to trigger LTD. This would not explain, however, the additional resistance of ascending axon synapses to CF-independent LTD nor the absence of postsynaptic LTP, which does not require such high levels of postsynaptic calcium elevation (Coesmans et al., 2004).

The temporal and spatial patterns of synaptic activation also influence the release of several factors involved in LTD and LTP induction, including NO, cGMP, and glutamate (Hartell et al., 2001; Marcaggi and Attwell, 2005; Namiki et al., 2005). Activation of spatially close synapses can cause glutamate spillover and activation of postsynaptic type 1 metabotropic glutamate receptors (mGluR1), leading to the production of endocannabinoids. These act retrogradely to limit NO and glutamate release and they have recently been shown to be involved in LTD (Safo and Regehr, 2005). It is conceivable, therefore, that direct stimulation of parallel fibers within the molecular layer might produce a relatively dense activation of synapses to a given PC than ascending axon activation, leading to higher levels of NO production, glutamate spillover, and mGluR1 activation (Marcaggi and Attwell, 2005), and consequently calcium release from intracellular stores (Finch and Augustine, 1998; Takechi et al., 1998). All of these 
elements are involved in LTD and presumably to some extent in postsynaptic LTP. If ascending axon inputs to a PC are more dispersed, one would predict that they are less able to trigger these pathways and consequently less likely to induce LTD or LTP. Synapse density would appear not to account for the selective plasticity at parallel fibers, however, because presynaptic and postsynaptic forms of LTP, as well as LTD, were evident at synapses activated by lateral stimulation of GCs, which is expected to produce a dispersed synaptic activation of PC by parallel fiber segments of the GC axon.

Differences in transmission properties between ascending axon and parallel fiber synapses, and their disparate abilities to undergo short- and longer-term forms of plasticity, may help to account for the limited spread of afferent information along parallel fibers. We suggest, therefore, that synapses formed by the two segments of the granule cell axon should be considered separately in experimental and theoretical studies of cerebellar cortical function.

\section{References}

Anderson WW, Collingridge GL (1999) A data acquisition program for online analysis of long-term potentiation and long-term depression. Soc Neurosci Abstr 23:665.

Anderson WW, Collingridge GL (2001) The LTP program: a data acquisition program for on-line analysis of long-term potentiation and other synaptic events. J Neurosci Methods 108:71-83.

Bekkers JM, Stevens CF (1990) Presynaptic mechanism for long-term potentiation in the hippocampus. Nature 346:724-729.

Bower JM, Woolston DC (1983) Congruence of spatial organization of tactile projections to granule cell and Purkinje cell layers of cerebellar hemispheres of the albino rat: vertical organization of cerebellar cortex. J Neurophysiol 49:745-766.

Coesmans M, Weber JT, De Zeeuw CI, Hansel C (2004) Bidirectional parallel fiber plasticity in the cerebellum under climbing fiber control. Neuron 44:691-700.

Cohen D, Yarom Y (1998) Patches of synchronized activity in the cerebellar cortex evoked by mossy-fiber stimulation: questioning the role of parallel fibers. Proc Natl Acad Sci USA 95:15032-15036.

Conquet F, Bashir ZI, Davies CH, Daniel H, Ferraguti F, Bordi F, Franz-Bacon K, Reggiani A, Matarese V, Conde F, Collingridge GL, Crepel F (1994) Motor deficit and impairment of synaptic plasticity in mice lacking mGluR1. Nature 372:237-243.

Eccles JC, Sabah NH, Schmidt RF, Taborikova H (1972) Integration by Purkyne cells of mossy and climbing fiber inputs from cutaneous mechanoreceptors. Exp Brain Res 15:498-520.

Finch EA, Augustine GJ (1998) Local calcium signalling by inositol-1,4,5trisphosphate in Purkinje cell dendrites. Nature 396:753-756.

Garwicz M, Andersson G (1992) Spread of synaptic activity along parallel fibres in cat cerebellar anterior lobe. Exp Brain Res 88:615-622.

Gundappa-Sulur G, De Schutter E, Bower JM (1999) Ascending granule cell axon: an important component of cerebellar cortical circuitry. J Comp Neurol 408:580-596.

Hartell NA (1994) Induction of cerebellar long-term depression requires activation of glutamate metabotropic receptors. NeuroReport 5:913-916.

Hartell NA (1996) Strong activation of parallel fibers produces localized calcium transients and a form of LTD that spreads to distant synapses. Neuron 16:601-610.
Hartell NA, Furuya S, Jacoby S, Okada D (2001) Intercellular action of nitric oxide increases cGMP in cerebellar Purkinje cells. NeuroReport 12:25-28.

Harvey RJ, Napper RM (1991) Quantitative studies on the mammalian cerebellum. Prog Neurobiol 36:437-463.

Heck D (1995) Sequential stimulation of guinea pig cerebellar cortex in vitro strongly affects Purkinje cells via parallel fibres. Naturwissenschaften 82:201-203.

Isope P, Barbour B (2002) Properties of unitary granule cell $\rightarrow$ Purkinje cell synapses in adult rat cerebellar slices. J Neurosci 22:9668-9678.

Jacoby S, Sims RE, Hartell NA (2001) Nitric oxide is required for the induction and heterosynaptic spread of cerebellar LTP. J Physiol (Lond) 535:825-839.

Jaeger D (2003) No parallel fiber volleys in the cerebellar cortex: evidence from cross-correlation analysis between Purkinje cells in a computer model and in recordings from anesthetized rats. J Comput Neurosci 14:311-327.

Kim J, Alger BE (2001) Random response fluctuations lead to spurious paired-pulse facilitation. J Neurosci 21:9608-9618.

Lev-Ram V, Wong ST, Storm DR, Tsien RY (2002) A new form of cerebellar long-term potentiation is postsynaptic and depends on nitric oxide but not cAMP. Proc Natl Acad Sci USA 99:8389-8393.

Lev-Ram V, Mehta SB, Kleinfeld D, Tsien RY (2003) Reversing cerebellar long-term depression. Proc Natl Acad Sci USA 100:15989-15993.

Llinás R (1982) General discussion: radial connectivity in the cerebellar cortex. A novel view regarding the functional organization of the molecular layer. In: The cerebellum: new vistas (Palay SL, Chan-Palay V, eds), pp 189-194. Berlin: Springer.

Marcaggi P, Attwell D (2005) Endocannabinoid signaling depends on the spatial pattern of synapse activation. Nat Neurosci 8:776-781.

Namiki S, Kakizawa S, Hirose K, Iino M (2005) NO signalling decodes frequency of neuronal activity and generates synapse-specific plasticity in mouse cerebellum. J Physiol (Lond) 566:849-863.

Napper RM, Harvey RJ (1988) Number of parallel fiber synapses on an individual Purkinje cell in the cerebellum of the rat. J Comp Neurol 274:168-177.

Pitchitpornchai C, Rawson JA, Rees S (1994) Morphology of parallel fibres in the cerebellar cortex of the rat: an experimental light and electron microscopic study with biocytin. J Comp Neurol 342:206-220.

Safo PK, Regehr WG (2005) Endocannabinoids control the induction of cerebellar LTD. Neuron 48:647-659.

Salin PA, Malenka RC, Nicoll RA (1996) Cyclic-AMP mediates a presynaptic form of LTP at cerebellar parallel fiber synapses. Neuron 16:797-803.

Santamaria F, Jaeger D, De Schutter E, Bower JM (2002) Modulatory effects of parallel fiber and molecular layer interneuron synaptic activity on Purkinje cell responses to ascending segment input: a modeling study. J Comput Neurosci 13:217-235.

Shibuki K, Okada D (1992) Cerebellar long-term potentiation under suppressed postsynaptic $\mathrm{Ca}^{2+}$ activity. NeuroReport 3:231-234.

Sims RE, Hartell NA (2005) Differences in transmission properties and susceptibility to long-term depression reveal functional specialization of ascending axon and parallel fiber synapses to Purkinje cells. J Neurosci 25:3246-3257.

Takechi H, Eilers J, Konnerth A (1998) A new class of synaptic response involving calcium release in dendritic spines. Nature 396:757-760.

Vranesic I, Iijima T, Ichikawa M, Matsumoto G, Knopfel T (1994) Signal transmission in the parallel fiber-Purkinje cell system visualized by highresolution imaging. Proc Natl Acad Sci USA 91:13014-13017.

Wang YT, Linden DJ (2000) Expression of cerebellar long-term depression requires postsynaptic clathrin-mediated endocytosis. Neuron 25:635-647. 